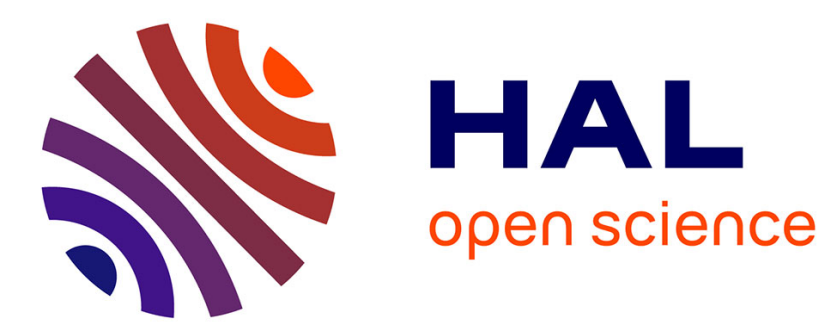

\title{
Optimal identification of delay-diffusive operators and application to the acoustic impedance of absorbent materials
}

Céline Casenave, Gérard Montseny

\section{- To cite this version:}

Céline Casenave, Gérard Montseny. Optimal identification of delay-diffusive operators and application to the acoustic impedance of absorbent materials. Topics in Time Delay Systems, Springer, pp.315325, 2009, 10.1007/978-3-642-02897-7_27 . hal-00833051

\section{HAL Id: hal-00833051 \\ https://hal.science/hal-00833051}

Submitted on 11 Jun 2013

HAL is a multi-disciplinary open access archive for the deposit and dissemination of scientific research documents, whether they are published or not. The documents may come from teaching and research institutions in France or abroad, or from public or private research centers.
L'archive ouverte pluridisciplinaire HAL, est destinée au dépôt et à la diffusion de documents scientifiques de niveau recherche, publiés ou non, émanant des établissements d'enseignement et de recherche français ou étrangers, des laboratoires publics ou privés. 


\title{
Optimal Identification of Delay-Diffusive Operators and Application to the Acoustic Impedance of Absorbent Materials
}

\author{
Céline Casenave ${ }^{1}$ and Gérard Montseny ${ }^{1}$ \\ ${ }^{1}$ LAAS-CNRS, University of Toulouse, 7 avenue du Colonel Roche 31077 Toulouse cedex \\ 4, France. casenave@laas. fr \\ 2 LAAS-CNRS, University of Toulouse, 7 avenue du Colonel Roche 31077 Toulouse cedex \\ 4, France. montseny@laas. fr
}

\begin{abstract}
Summary. We present an original method devoted to the optimal identification of a wide class of complex linear operators involving some delay components, based on suitable infinite dimensional state formulations of diffusive type. Thanks to the intrinsic properties of these state formulations, cheap and precise numerical approximations are straightforwardly obtained, leading to approximate quadratic problems of reasonable dimension. We then propose this method for identification of the acoustic impedance of absorbent materials designed for noise reduction of aircraft motors.
\end{abstract}

\section{Introduction}

Identification of dynamic input-output systems is a central problem each time the knowledge of the process under consideration is too imprecise to get sufficiently accurate models from physical analysis (note anyway that such an analysis is sometimes impossible to perform because too complex). However, identification problems can also present some serious difficulties. Namely, when the input-output operator under consideration involves distributed underlying phenomena, significant non rational components, which are associated to infinitedimensional realizations in the time domain, are quasi systematically generated.

When the operator to be identified is linear, a convenient and rather general approach consists in working in the frequency domain. So, any causal operator can be well-defined by its symbol $Q(t, i \omega)$, that is the Fourier transform of the so-called impulse response $h(t, s)$ of the operator. In the case of convolution operators, the symbol no more depends on $t$ and the problem of identifying $Q(i \omega)$ can be classically solved from physical measurements by means of Fourier techniques. Note however that purely frequency identification presents some well-known shortcomings. In particular, the so-identified symbol is in general ill adapted to the construction of efficient time-realizations. This is partly due to excessive numerical cost of quadrature approximations resulting from the intrinsic convolutive nature of the associated operator, sometimes with long memory [2] or even delay-like behaviors [10]. Another heavy shortcoming is that frequency methods are incompatible with real-time identification (and so with pursuit when the symbol has the ability to evolve slowly). In opposite, time domain 
identification techniques do not present such drawbacks. However, their scope is in general not so wide. See for example [8] for an interesting optimal method based on ARMA lattices.

Nevertheless, the notion of symbol can remain suitable for temporal identification, as it will be shown in this study. The proposed identification method is based on the so-called diffusive representation theory [9] and was previously implemented, in a simpler version, on various problems [2], [4]. According to the diffusive representation approach, state formulations of diffusive type are available for a wide class of integral causal operators (including both rational and non rational ones) and the identification problem is carried over the socalled $\gamma$-symbol, defined as a suitable function (of an auxiliary real variable) easily deduced from the classical symbol. This $\gamma$-symbol entirely characterizes the associated operator, up to a suitable infinite-dimensional state equation to be chosen a priori. In any case, cheap and precise finite-dimensional approximations of this state representation can be straightforwardly built, running into finite-dimensional problems easy to solve by means of standard techniques. Among the numerous advantages of this approach, we can mention in particular:

- a stable differential input-output time-formulation is available as soon as the $\gamma$-symbol is well-defined;

- recursive identification algorithms are easily built and implemented, allowing real-time identification or even pursuit (in that case, the $\gamma$-symbol is depending on $t$ );

- similarly to purely frequency methods, no qualitative difference is made between rational and non rational components which can then be identified by the same process.

The paper is organized as follows. In section 2 we first introduce a few fundamental notions of the diffusive representation theory. In section 3 we describe the identification method under consideration, based on the previously introduced notions. We then consider in section 4 a typical problem whose difficulty lies in the fact that both diffusive and delay components are involved but are not separable, and to which the diffusive representation approach is welladapted: the identification of the acoustic impedance of an absorbent material devoted to aircraft motors noise reduction. Finally, a significant numerical example is given in section 5

\section{Diffusive formulation of causal integral operators}

In this section, we present a simplified version of a general methodology introduced and developed in [9] in a general framework.

We consider a causal operator defined, on any continuous function $u: \mathbb{R}^{+} \rightarrow \mathbb{R}$, by:

$$
u \mapsto \int_{0}^{t} h(t-s) u(s) d s .
$$

We denote $H$ the Laplace transform of $h$ and $H\left(\partial_{t}\right)$ the convolution operator defined by (1).

Let $u^{t}(s)=\mathbf{1}_{]-\infty, t]}(s) u(s)$ the restriction of $u$ to its past and $u_{t}(s)=u^{t}(t-s)$ the history of $u$. From causality of $H\left(\partial_{t}\right)$, we deduce:

$$
\left(H\left(\partial_{t}\right)\left(u-u^{t}\right)\right)(t)=0 \text { for all } t
$$

then, we have for any continuous function $u$ :

$$
\left(H\left(\partial_{t}\right) u\right)(t)=\left[\mathcal{L}^{-1}(H \mathcal{L} u)\right](t)=\left[\mathcal{L}^{-1}\left(H \mathcal{L} u^{t}\right)\right](t) .
$$

We define:

$$
\Psi_{u}(t, p):=\mathrm{e}^{p t}\left(\mathcal{L} u^{t}\right)(p)=\left(\mathcal{L} u_{t}\right)(-p) ;
$$

by computing $\partial_{t}\left(\mathcal{L} u_{t}\right)$, Laplace inversion and use of $[2]$, it can be shown: 
Lemma 1. 1. The function $\Psi_{u}$ is solution of the differential equation:

$$
\partial_{t} \Psi(t, p)=p \Psi(t, p)+u, \quad t>0, \quad \Psi(0, p)=0 .
$$

2. For any $b \geqslant 0$,

$$
\left(H\left(\partial_{t}\right) u\right)(t)=\frac{1}{2 i \pi} \int_{b-i \infty}^{b+i \infty} H(p) \Psi_{u}(t, p) d p
$$

We denote $\Omega$ the holomorphic domain of $H$ (after analytic continuation). Let $\gamma$ a simple arc in $\mathbb{C}^{-}$, closed at infinity and separating $\mathbb{C}$ in two open domains denoted $\Omega_{\gamma}^{+}$and $\Omega_{\gamma}^{-}$such that $\Omega_{\gamma}^{-} \subset \mathbb{C}^{-}$. By use of standard techniques (Cauchy theorem, Jordan lemma [6]), it can be shown:

Lemma 2. For $\gamma \subset \Omega$ such that $H$ is holomorphic in $\Omega_{\gamma}^{+}$, if $H(p) \rightarrow 0$ when $p \rightarrow \infty$ in $\Omega_{\gamma}^{+}$, then:

$$
\left(H\left(\partial_{t}\right) u\right)(t)=\frac{1}{2 i \pi} \int_{\tilde{\gamma}} H(p) \Psi_{u}(t, p) d p,
$$

where $\tilde{\gamma}$ is any closed ${ }^{3}$ simple arc in $\Omega_{\gamma}^{+}$such that $\gamma \subset \Omega_{\tilde{\gamma}}^{-}$.

We now suppose that $\gamma$ and $\tilde{\gamma}$ are defined by functions of the Sobolev space $\Phi^{4} W_{\text {loc }}^{1, \infty}(\mathbb{R} ; \mathbb{C})$, also denoted $\gamma, \tilde{\gamma}$. We use the convenient notation $\langle\mu, \psi\rangle=\int \mu \psi d \xi$.

Under hypothesis of lemma2] we have [9]:

Theorem 1. If the possible singularities of $H$ on $\gamma$ are simple poles or branching points such that $|H \circ \gamma|$ is locally integrable in their neighborhood, then:

1. with $\tilde{\mu}=\frac{\tilde{\gamma}^{\prime}}{2 \mathrm{i} \pi} H \circ \tilde{\gamma}$ and $\tilde{\psi}(t,)=.\Psi_{u}(t,.) \circ \tilde{\gamma}$ :

$$
\left(H\left(\partial_{t}\right) u\right)(t)=\langle\tilde{\mu}, \tilde{\psi}(t, .)\rangle
$$

2. with $h^{5} \tilde{\gamma}_{n} \rightarrow \gamma$ in $W_{\mathrm{loc}}^{1, \infty}$ and $\mu=\frac{\tilde{\gamma}^{\prime}}{2 \mathrm{i} \pi} \lim H \circ \tilde{\gamma}_{n}$ in the sense of measures:

$$
\left(H\left(\partial_{t}\right) u\right)(t)=\langle\mu, \psi(t, .)\rangle
$$

where $\psi(t, \xi)$ is solution of the following Cauchy problem on $(t, \xi) \in \mathbb{R}^{*+} \times \mathbb{R}$ :

$$
\partial_{t} \psi(t, \xi)=\gamma(\xi) \psi(t, \xi)+u(t), \psi(0, \xi)=0 .
$$

Definition 1. The measure $\mu$ defined in theorem $\square$ is called the $\gamma$-symbol of operator $H\left(\partial_{t}\right)$.

Such a formulation can be extended to operators of the form $\partial_{t} \circ H\left(\partial_{t}\right)$ where $H\left(\partial_{t}\right)$ verifies the hypothesis of theorem 1 We indeed have (formally):

$$
\left\{\begin{array}{l}
\partial_{t} \psi(t, \xi)=\gamma(\xi) \psi(t, \xi)+u(t), \psi(0, \xi)=0 \\
\partial_{t}\left(H\left(\partial_{t}\right) u\right)(t)=\langle\mu, \gamma \psi(t, .)+u(t)\rangle
\end{array}\right.
$$

\footnotetext{
${ }^{3}$ Possibly at infinity

${ }^{4} W_{\text {loc }}^{1, \infty}(\mathbb{R} ; \mathbb{C})$ is the topological space of measurable functions $f: \mathbb{R} \rightarrow \mathbb{C}$ such that $f, f^{\prime} \in L_{\mathrm{loc}}^{\infty}$ (that is $f$ and $f^{\prime}$ are locally bounded in the almost everywhere sense).

${ }^{5}$ This convergence mode means that $\tilde{\gamma}_{n_{\mid K}}-\gamma_{\mid K} \rightarrow 0$ and $\tilde{\gamma}_{n_{\mid K}}^{\prime}-\gamma_{\mid K}^{\prime} \rightarrow 0$ uniformly on any bounded set $K$.
} 
The state equation (8) is infinite-dimensional. To get numerical approximations, we consider a discretization $\left(\xi_{k}\right)_{k=1: n}$ of the variable $\xi$ and approximations $\mu_{n}$ of the $\gamma$-symbol $\mu$ defined by atomic measures such as:

$$
\mu_{n}=\sum_{k=1}^{n} \mu_{n}^{k} \delta_{\xi_{k}} .
$$

Let us denote $\mathcal{M}_{n}$ the space of atomic measures on the mesh $\left\{\xi_{k}\right\}_{k=1: n}$. If $\cup_{n} \mathcal{M}_{n}$ is dense in the space of measures, we have [9]:

$$
\left\langle\mu_{n}, \psi\right\rangle \underset{n \rightarrow+\infty}{\longrightarrow}\langle\mu, \psi\rangle, \forall \psi \in \mathcal{C}^{0}(\mathbb{R}) .
$$

So we have the finite-dimensional approximate state formulation of $\partial_{t} \circ H\left(\partial_{t}\right)$ :

$$
\left\{\begin{array}{l}
\partial_{t} \psi\left(t, \xi_{k}\right)=\gamma\left(\xi_{k}\right) \psi\left(t, \xi_{k}\right)+u(t), k=1: n \\
\psi\left(0, \xi_{k}\right)=0 \\
\partial_{t}\left(H\left(\partial_{t}\right) u\right)(t) \simeq \sum_{k=1}^{n} \gamma\left(\xi_{k}\right) \mu_{n}^{k} \psi\left(t, \xi_{k}\right)+\left(\sum_{k=1}^{n} \mu_{n}^{k}\right) u(t)
\end{array}\right.
$$

Note that when operator $H\left(\partial_{t}\right)$ is not pseudo-differential [11], namely when some delay-type behaviors are present, the function $\gamma$ must verify [10]:

$$
\begin{aligned}
& \lim _{\xi \rightarrow \pm \infty} \operatorname{Im}(\gamma(\xi))= \pm \infty \\
& \text { and } \exists a<0, \operatorname{Re}(\gamma(\xi)) \geqslant a .
\end{aligned}
$$

\section{Optimal identification of the $\gamma$-symbol}

In this section, we focus on the problem of identification of a convolution operator:

$$
u \longmapsto y=H\left(\partial_{t}\right) u,
$$

where the symbol $H(p)$ is supposed to verify the hypothesis of theorem 1 For simplicity, we present formal developments only; more details will be given in a further paper.

We denote by $\mu$ the $\gamma$-symbol of $H\left(\partial_{t}\right)$, as defined in section 2 The problem then consists of identifying $\mu$ from the data $u$ and $y^{*}$, this last term denoting the measurement of $y$, in general sullied by some additive noise.

Given a suitable $\gamma$, we consider the diffusive realization 817] of $H\left(\partial_{t}\right)$ and we denote $\psi_{u}$ the solution of 8 . By defining $A_{u}$ the following linear operator:

$$
A_{u}: \mu \mapsto\left\langle\mu, \psi_{u}\right\rangle,
$$

we obviously have:

$$
y=A_{u} \mu .
$$

Given suitable Hilbert spaces $\mathcal{E}, \mathcal{F}$ (not specified here), we consider the problem:

$$
\min _{\mu \in \mathcal{E}}\left\|A_{u} \mu-y^{*}\right\|_{\mathcal{F}}^{2},
$$

the solution of which is classically given by: 


$$
\mu^{*}=A_{u}^{\dagger} y^{*}
$$

where $A_{u}^{\dagger}$ denotes the pseudo-inverse of $A_{u}[1]$. So, in the sense of the hilbertian norm of $\mathcal{F}$, the estimation $\mu^{*}$ of $\mu$ is optimal.

$>$ From the numerical point of view, after suitable time discretization and approximation of $\mu$ such as described in section 2 we have:

$$
\left(A_{u} \mu\right)_{i}=\sum_{k=1}^{n} \mu_{n}^{k} \psi\left(t_{i}, \xi_{k}\right) ;
$$

then the operator $A_{u}$ can be expressed as a matrix $\left(\psi_{u}\left(t_{i}, \xi_{k}\right)\right)_{i=1: m, k=1: n}$ and its pseudoinverse is classically expressed (with $m \gg n$ ):

$$
A_{u}^{\dagger}=\left(A_{u}^{*} A_{u}+\epsilon I\right)^{-1} A_{u}^{*} .
$$

Remark 1. Recursive formulations of [12) can be established under the form (see [4]):

$$
\mu_{t}^{*}=\mu_{t-\Delta t}^{*}+K_{t-\Delta t}\left(y^{*}-A_{u} \mu_{t-\Delta t}^{*}\right)_{\mid[0, t]} ;
$$

such formulations allow real-time pursuit of $\mu$ in case of slowly varying operators $Q\left(t, \partial_{t}\right)$.

\section{Application to the impedance of an absorbent material}

First, let us present the physical problem under consideration.

In order to reduce the noise generated by aircraft motors, the use of absorbent coverings is an efficient solution. For particular cases where stringent thermal or mechanical constraints are present, such as in hot zones of motors, porous metallic materials have been proposed and studied [5], [7]. Due to the complex structure of such materials, the generated acoustic impedances are non rational complex functions of the frequency with unavoidable delay behaviors due to the presence of propagating waves in such media. In addition, their analytic determination is difficult and possibly poorly reliant. So, experimental measurements followed by identification processes remain necessary when accurate models are expected to carry out numerical simulations. In this way, it becomes possible, for a given material, to numerically compute the aeroacoustic field in a motor cavity equipped with absorbent walls, in aim of evaluating the amount of noise reduction.

Fortunately, the impedance of an homogeneous 2D coating does not depend on the surface parameterization variables and therefore, at least for sufficiently high frequencies, it can be identified from physical measurements relating to a small sample of the material (see figure 11. In the sequel, we show how the method introduced in section 4 can be implemented on such a problem.

For simplicity of the analysis, we suppose that the problem described in figure 1 can be reduced to a 1D problem, which is legitimate if the source and the sensor are sufficiently far from the absorbent sample. This will be sufficient to introduce the principle of the method. For a more realistic analysis, the 3D problem can be similarly treated up to technical adaptations.

Under these conditions, the absorbent material sample can be summarized by an impedance operator $Q\left(\partial_{t}\right)$ at point $x=1$. The $1 \mathrm{D}$ problem of acoustic waves propagation can then be written a:

\footnotetext{
${ }^{6}$ As usual, $\epsilon$ is a small positive parameter devoted to numerical conditioning.

${ }^{7}$ For simplicity the celerity has been taken equal to 1 .
} 


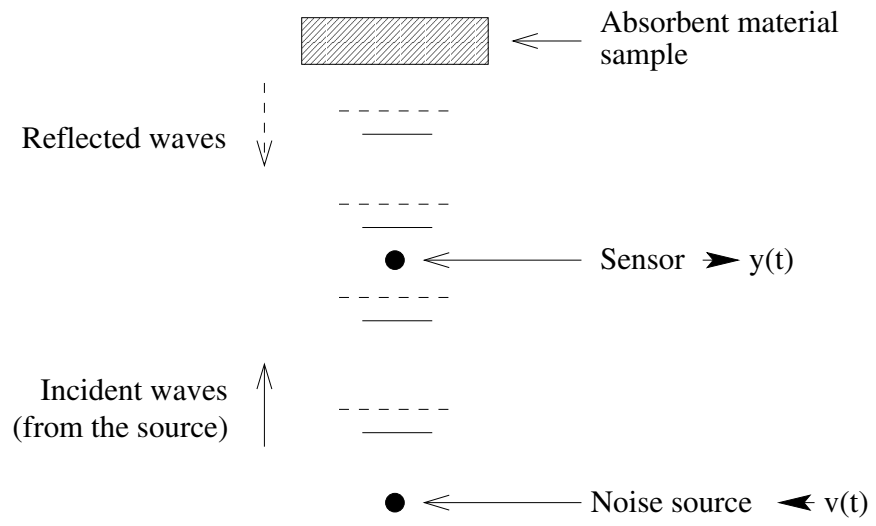

Fig. 1. Physical measurement for the identification of the impedance of an homogeneous absorbing material.

$$
\left.\left.\partial_{t}^{2} P=\partial_{x}^{2} P+v \otimes \delta_{0} \quad \text { on } x \in\right]-\infty, 1\right],
$$

where $P$ is the acoustic pressure and $v \otimes \delta_{0}$ denotes the source at point $x=0$, with $v(t)$ a known time-function. We have:

$$
\partial_{x} P_{\mid x=1}=Q\left(\partial_{t}\right) \partial_{t} P_{\mid x=1} .
$$

Finally the acoustic pressure is measured at a point $x_{m}$ :

$$
y=P\left(t, x_{m}\right) .
$$

Then, the identification problem is to build an approximation of operator $Q\left(\partial_{t}\right)$ from the data $y$ and $v$ (note that in practice, the measurement $y$ can be perturbed by an additive noise, not explicitly specified here).

Let us compute the expression of the operator $P(., 0) \mapsto y$, denoted by $K\left(\partial_{t}\right)$. For that, we must compute the reflection coefficient at $x=1$ for any frequency $\omega$. Let $\varphi$ a harmonic solution of (13) with $v=0$. It can be expressed as $\varphi=\varphi^{i}+\varphi^{r}$ where $\varphi^{i}$ denotes an incident wave (propagating in the direction of increasing $x$ ) and $\varphi^{r}$ the associated reflected wave (propagating in the sense of decreasing $x$ ), respectively given by:

$$
\begin{aligned}
\varphi^{i}(t, \omega, x) & =e^{i \omega(t-x)}, \\
\varphi^{r}(t, \omega, x) & =k(\omega) e^{i \omega(t+x)},
\end{aligned}
$$

where $k(\omega)$ is the reflection coefficient at frequency $\omega$, depending on $Q(i \omega)$. At point $x=1$, the coefficient $k(\omega)$ verifies the impedance relation 14 which leads after simple computations to:

So we have:

$$
k(\omega)=\frac{1+Q(i \omega)}{1-Q(i \omega)} e^{-2 i \omega}
$$

$$
\varphi(t, \omega, x)=\left(e^{-i \omega x}+\frac{1+Q(i \omega)}{1-Q(i \omega)} e^{i \omega(x-2)}\right) e^{i \omega t},
$$


and then (from 15b):

$$
K(i \omega)=e^{-i \omega x_{m}}+\frac{1+Q(i \omega)}{1-Q(i \omega)} e^{i \omega\left(x_{m}-2\right)} .
$$

On the other hand, the solution of 13 is classically given by:

$$
P(t, x)=\frac{1}{2}\left(\partial_{t}^{-1} v\right)(t-x) ;
$$

by harmonic synthesis, the input-output relation $v \mapsto y$ can therefore be made explicit in the time domain $\left(\tau_{a}\right.$ denotes the translation operator $f(t) \mapsto f(t-a)$ ):

$$
y=\frac{1}{2} \tau_{x_{m}} \circ \partial_{t}^{-1} v+\frac{1}{2} M\left(\partial_{t}\right) \circ \tau_{2-x_{m}} \circ \partial_{t}^{-1} v
$$

with:

$$
M(i \omega):=\frac{1+Q(i \omega)}{1-Q(i \omega)}
$$

By defining the new data and notations:

$$
\begin{gathered}
\tilde{y}:=y-\frac{1}{2}\left(\tau_{x_{m}}+\tau_{2-x_{m}}\right) \circ \partial_{t}^{-1} v, \\
\tilde{v}:=\frac{1}{2} \tau_{2-x_{m}} v, \\
\widetilde{M}\left(\partial_{t}\right):=M\left(\partial_{t}\right)-1,
\end{gathered}
$$

we obtain the suitable relation:

$$
\tilde{y}=\partial_{t}^{-1} \circ \widetilde{M}\left(\partial_{t}\right) \tilde{v}
$$

on which we can directly apply the method presented in section 3 to identify the associated $\gamma$-symbol.

Let $\mu$ denote indifferently the so-identified $\gamma$-symbol or the exact $\gamma$-symbol of $\partial_{t}^{-1}$ 。 $\widetilde{M}\left(\partial_{t}\right)$. By considering the following relation deduced from 17 :

$$
Q(i \omega)=\frac{\widetilde{M}(i \omega)}{\widetilde{M}(i \omega)+2}
$$

and from elementary computations, we then deduce the following input-output state representation of operator $w \mapsto Q\left(\partial_{t}\right) w$ :

$$
\left\{\begin{array}{l}
\partial_{t} \psi=\gamma \psi-\frac{1}{2} \frac{\langle\gamma \mu, \psi\rangle}{\langle\mu, 1\rangle+1}+\frac{1}{\langle\mu, 1\rangle+1} w, \psi_{0}=0 \\
Q\left(\partial_{t}\right) w=\frac{1}{2} \frac{\langle\gamma \mu, \psi\rangle}{\langle\mu, 1\rangle+1}+\frac{\langle\mu, 1\rangle}{\langle\mu, 1\rangle+1} w
\end{array}\right.
$$

which is exact (infinite-dimensional) if $\mu$ is the exact $\gamma$-symbol, and approximate (finitedimensiona 8 ) if $\mu$ is the identified $\gamma$-symbol, of the form (10).

Remark 2. A more precise analysis can be performed by replacing (13) by the 3D problem: $\partial_{t}^{2} P=\Delta P+v \otimes \delta_{0}$. By use of Green functions, similar (but more technical) computations can be achieved and (18) is obtained again while the expression of $\tilde{y}$ and $\tilde{v}$ involves more complex (but explicitly known) operators derived from the Green functions.

$\overline{{ }^{8} \text { In that case }},\langle f, g\rangle=\sum_{k} f_{k} g_{k}$. 


\section{Numerical example and conclusion}

We consider the porous material studied in [7], [5], the impedance of which has been analytically computed in [3]. This impedance is expressed:

$$
Q(i \omega)=\varphi \sqrt{\frac{\chi_{\mathrm{eff}}(i \omega)}{\rho_{\mathrm{eff}}(i \omega)}} \tanh \left(i \omega \sqrt{\chi_{\mathrm{eff}}(i \omega) \rho_{\mathrm{eff}}(i \omega)}\right),
$$

where $\varphi=3.33$ and the functions $\rho_{\mathrm{eff}}(i \omega)$ and $\chi_{\mathrm{eff}}(i \omega)$ are respectively the so-called effective density of Pride et al. and the effective compressibility of Lafarge. These functions are expressed [5]:

with

$$
\begin{aligned}
& \rho_{\mathrm{eff}}(i \omega)=e \rho\left(1+a \frac{(1+b i \omega)^{\frac{1}{2}}}{i \omega}\right) \\
& \chi_{\mathrm{eff}}(i \omega)=e \chi\left(1-c \frac{i \omega}{i \omega+a^{\prime}\left(1+b^{\prime} i \omega\right)^{\frac{1}{2}}}\right),
\end{aligned}
$$

$$
\begin{gathered}
\rho=\rho_{0} \alpha_{\infty}, \chi=\frac{1}{P_{0}}, a=\frac{8 \mu}{\rho_{0} \Lambda^{2}}, a^{\prime}=\frac{8 \mu}{\rho_{0} \Lambda^{\prime 2}}, \\
b=\frac{1}{2 a}, b^{\prime}=\frac{1}{2 a^{\prime}}, 0<c=\frac{\gamma-1}{\gamma}<1,
\end{gathered}
$$

where $e, \rho_{0}, P_{0}, \mu, \gamma, \alpha_{\infty}, \Lambda, \Lambda^{\prime}$ are physical parameters with values $\Lambda=\Lambda^{\prime}=0.110^{-3} \mathrm{~m}$, $\rho_{0}=1.2 \mathrm{~kg} \cdot \mathrm{m}^{-3}, P_{0}=10^{5} \mathrm{~Pa}, \mu=1.810^{-5} \mathrm{~kg} \cdot \mathrm{m}^{-1} \cdot \mathrm{s}^{-1}, \gamma=1.4, \alpha_{\infty}=1.3, e=$ $510^{-2} \mathrm{~m}$.

$>$ From standard analysis, it can be shown that the analytic continuation of $Q$ presents singularities which can be asymptotically localized near a vertical straight line (see figure 2): operator $Q\left(\partial_{t}\right)$ presents some underlying behaviors of delay type, inherited from propagative modes inside the porous material and from which result complex magnitude and phase variations of $Q$, making the identification problem possibly sensitive or even ill-posed.

In aim of highlighting the efficiency of the previously introduced method, we consider the problem of numerical identification of such an operator $Q\left(\partial_{t}\right)$ under its state formulation (19) in the ideal situation described in section 4

The measurement data $y^{*}=y+\varepsilon$ have been generated on $t \in[0, T]$ from accurate numerical simulation of 16, with $v$ a gaussian white noise and $\varepsilon$ an output gaussian white noise with signal-to-noise ratio equal to $26 \mathrm{~dB}$.

The numerical parameters are $\Delta t=10^{-5} \mathrm{~s}, T=0.05 \mathrm{~s}, \xi_{\min }=\xi_{1}=10^{3} \mathrm{rad} / \mathrm{s}, \xi_{\max }=$ $\xi_{n}=310^{5} \mathrm{rad} / \mathrm{s}, \xi_{k+1}=r \xi_{k}, k=1: n-1, n=100, \gamma(\xi)=|\xi| \cos \alpha+i \xi \sin \alpha$, $\alpha=100^{\circ}$.

The comparison between the exact and identified frequency responses relating to the impedance $Q(i \omega)$ is available in figures 3 and 4 As expected, the identification is accurate in the frequency band $\left[10^{3} \mathrm{rad} / \mathrm{s}, 310^{5} \mathrm{rad} / \mathrm{s}\right]$ covered by the set $\left\{\gamma\left(\xi_{k}\right)\right\}_{k=1: n}$. We can note that identification remains correct on the whole audio-frequency band, namely at low frequencies thanks to the asymptotically rational nature of $Q(i \omega)$ which behaves like $i \omega$ when $\omega \rightarrow 0$.

In figure 5, we can see the graph of the identified $\gamma$-symbol associated to operator $\partial_{t}^{-1} \circ$ $\widetilde{M}\left(\partial_{t}\right)$ and from which the operator $Q\left(\partial_{t}\right)$ is realized via the input-output formulation [19].

Beyond these quantitative results, we can add that this identification method is robust with respect to the level of the perturbation noise $\varepsilon$, the time of measurements $T$ and the dimension $n$ of the identified model. This last essential property is a consequence of the

\footnotetext{
${ }^{9}$ Up to the numerical simulation errors, which are negligible in the frequency band under consideration.
} 


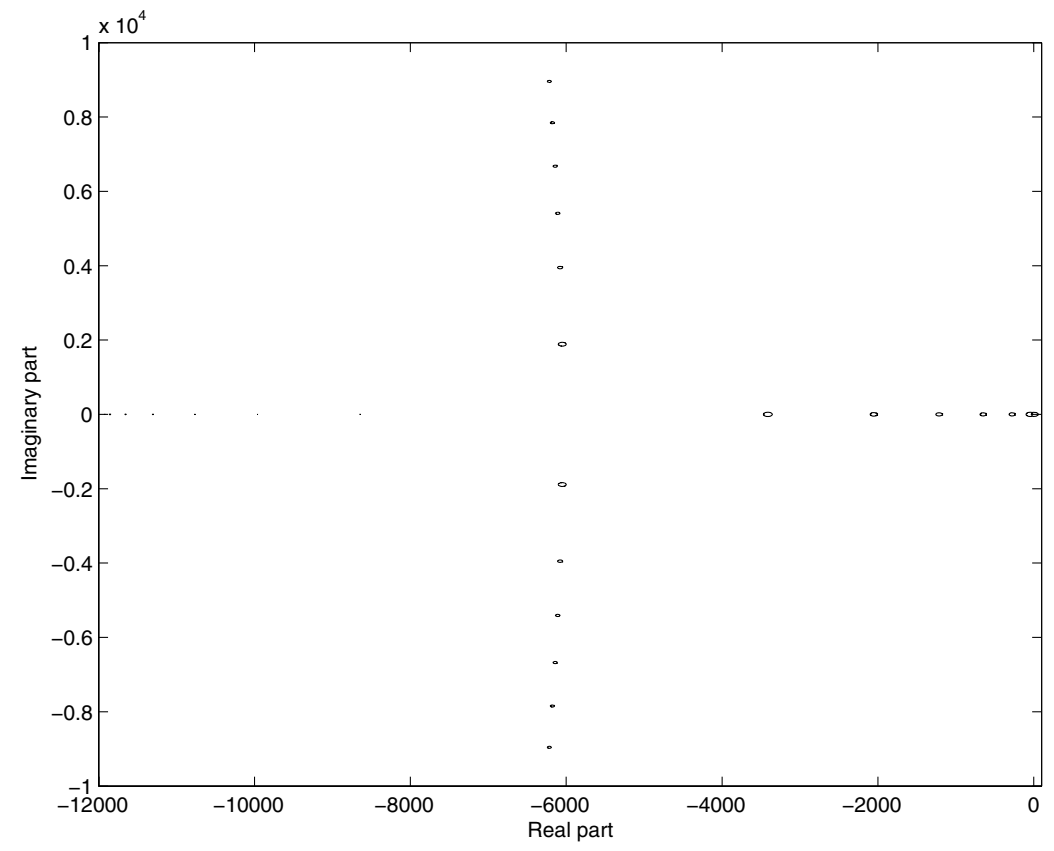

Fig. 2. Singularities of the symbol $Q(p)$.

fact that the poles $\gamma\left(\xi_{k}\right)$ of the identified transfer function are imposed a priori by the $\xi$ discretization of a suitable diffusive input-output model (namely 9) mathematically wellposed and able to realize a wide class of operators in which belongs the non rational operator under consideration. It follows, thanks to the properties of diffusive representation, that the identified $\gamma$-symbol $\mu$ converges (in a sense not precised here), when $n \rightarrow+\infty, \alpha \rightarrow \frac{\pi^{+}}{2}$, $\xi_{\min } \rightarrow 0^{+}, \xi_{\max } \rightarrow+\infty$ and $T \rightarrow+\infty$, to the exact one, and so for the identified impedance $Q(i \omega)$ in the sense of the Fréchet space $L_{\text {loc }}^{2}\left(\mathbb{R}_{\omega}\right)$.

\section{References}

1. Ben-Israel, A., Greville, T.N.E.: Generalized inverses: theory and applications. Springer, New York (2003)

2. Bidan, P., Lebey, T., Montseny, G., Neacsu, C., Saint-Michel, J.: Transient voltage distribution in motor windings fed by inverter: experimental study and modeling. IEEE Trans. on Power Electronics 16(1), 92-100 (2001)

3. Casenave, C., Montseny, E.: Dissipative state formulations and numerical simulation of a porous medium for boundary absorbing control of aeroacoustic waves. In: Proc. 17th IFAC World Congress, Seoul, Korea, July 6-11 (2008)

4. Garcia, G., Bernussou, J.: Identification of the dynamics of a lead acid battery. In: ESAIM: Proceedings, p. 5 (1998)

5. Gasser, S.: Etude des propriétés acoustiques et mécaniques d'un matériau métallique poreux à base de sphères creuses de nickel. PhD Thesis, Grenoble, France (2003) 


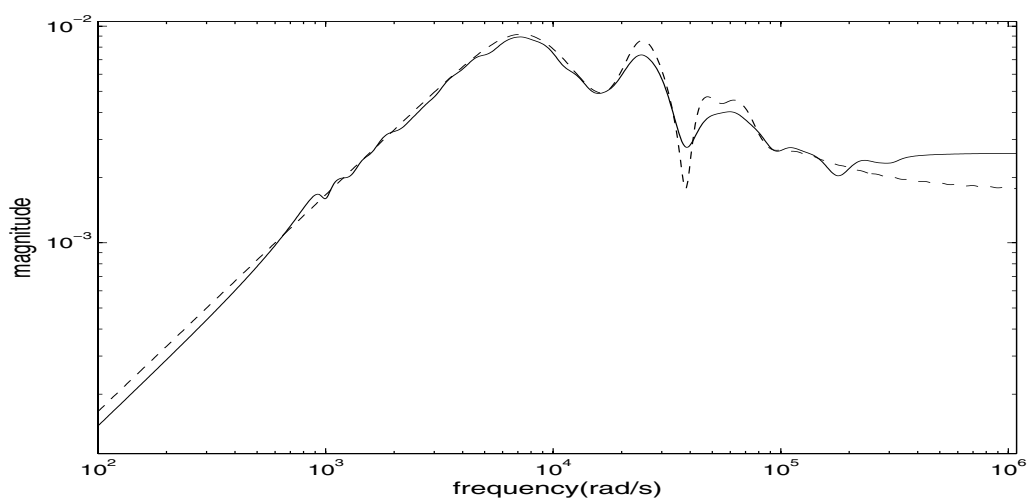

Fig. 3. Identified (-) and exact (- - -) frequency responses of operator $Q\left(\partial_{t}\right)$.

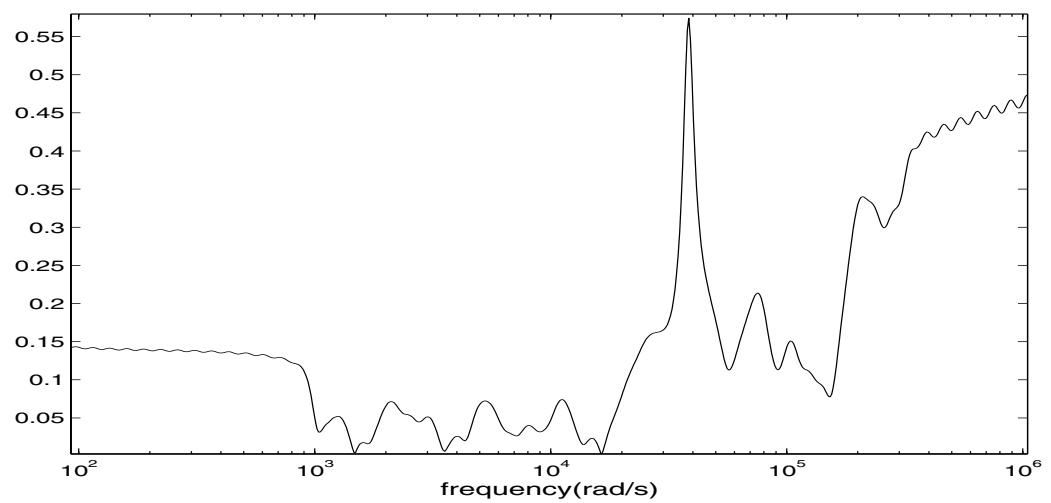

Fig. 4. Relative identification error $\left|\frac{Q-Q^{*}}{Q}\right|$.

6. Lavrentiev, M., Chabat, B.: Méthodes de la théorie des fonctions d'une variable complexe, MIR edn., Moscow, Russia (1977)

7. Mazet, P.A., Ventribout, Y.: Control of Aero-acoustic Propagations with Wall Impedance Boundary Conditions: Application to a Porous Material Model. In: WAVES 2005, Providence, USA (2005)

8. Monin, A., Salut, G.: ARMA Lattice identification: a new hereditary algorithm. IEEE Transactions on Signal Processing 44(2), 360-370 (1996)

9. Montseny, G.: Représentation diffusive. Hermes-Science, Paris, France (2003)

10. Montseny, G.: Diffusive representation for operators involving delays. In: Loiseau, J.J., Chiasson, J. (eds.) Applications of time-delay systems, pp. 217-232. Springer, Heidelberg (2007)

11. Taylor, M.E.: Partial differential equations - II. Applied Mathematical Sciences, p. 116. Springer, Heidelberg (1997) 


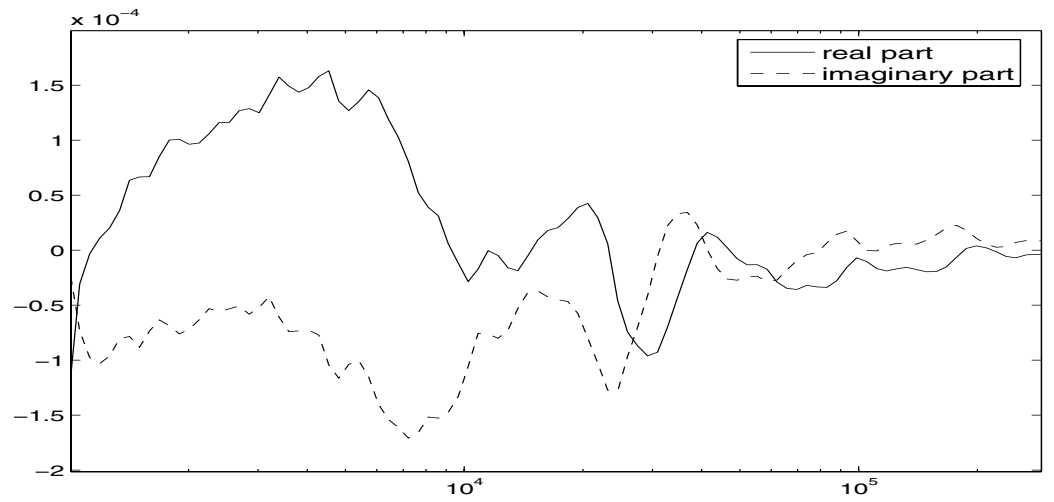

Fig. 5. Identified $\gamma$-symbol $\mu(\xi), \xi>0$. 